\title{
MULTIFREQUENCY POLARIZATION VARIATIONS IN 0917+624
}

\author{
S.J. QIAN, A. KRAUS, T.P. KRICHBAUM, A. WITZEL and J.A. ZENSUS \\ Max-Planck-Institut für Radioastronomie, Bonn, Germany
}

\section{Introduction}

Intraday variability in compact flat-spectrum radio sources has been intensively studied in recent years. For most IDV events the apparent brightness temperatures derived from the observed timescales are in the range of $T_{b, a p p} \sim 10^{16-18} \mathrm{~K}$. For extremely rapid variations, $T_{b, a p p}$ can reach up to $\sim 10^{21} \mathrm{~K}$ (e.g. Kedziora-Chudczer et al., 1997). Refractive interstellar scintillation may be the most likely extrinsic mechanism (Rickett et al., 1995; Qian, 1994a; Qian, 1994b). Especially for the case of extreme $T_{b, c i p p}\left(>10^{18} \mathrm{~K}\right.$ ) RISS may be dominant (Dennet-Thorpe and de Bruyn, 2000). However, some IDV events with $T_{a p p} \sim 10^{17-18} \mathrm{~K}$ show evidence for an intrinsic origin, e.g. the correlated radio-optical intraday variations observed in the BL Lac object 0716+714 (Wagner and Witzel, 1995, Qian et al., 1996). It seems important to distinguish between IDV which is a phenomenon intrinsic to the compact radio sources and IDV which is primarily due to RISS. Multifrequency polarization and VLBI observations would be most helpful (Gabuzda and Kochanev, 1997).

\section{TABLE I}

May89-event $(0917+624)$ : the mean values of the observed total flux density, polarized flux density, polarization angle, polarization degree and the modulation indices

\begin{tabular}{lcccccc}
\hline$\lambda(\mathrm{cm})$ & $\bar{I}(\mathrm{Jy})$ & $\bar{P}(\mathrm{mJy})$ & $\bar{\chi}\left({ }^{\circ}\right)$ & $\bar{p}(\%)$ & $m_{I}(\%)$ & $m_{P}(\%)$ \\
\hline 20 & 1.14 & 28.5 & 36.3 & 2.49 & 4.0 & 8.3 \\
11 & 1.36 & 11.6 & 27.1 & 0.85 & 6.0 & 28.8 \\
6 & 1.52 & 15.6 & -5.8 & 1.03 & 3.8 & 18.8 \\
3.6 & 1.57 & 18.0 & -18.9 & 1.15 & 2.0 & 8.8 \\
2 & 1.51 & 25.6 & -18.9 & 1.70 & 1.4 & 8.6 \\
\hline
\end{tabular}



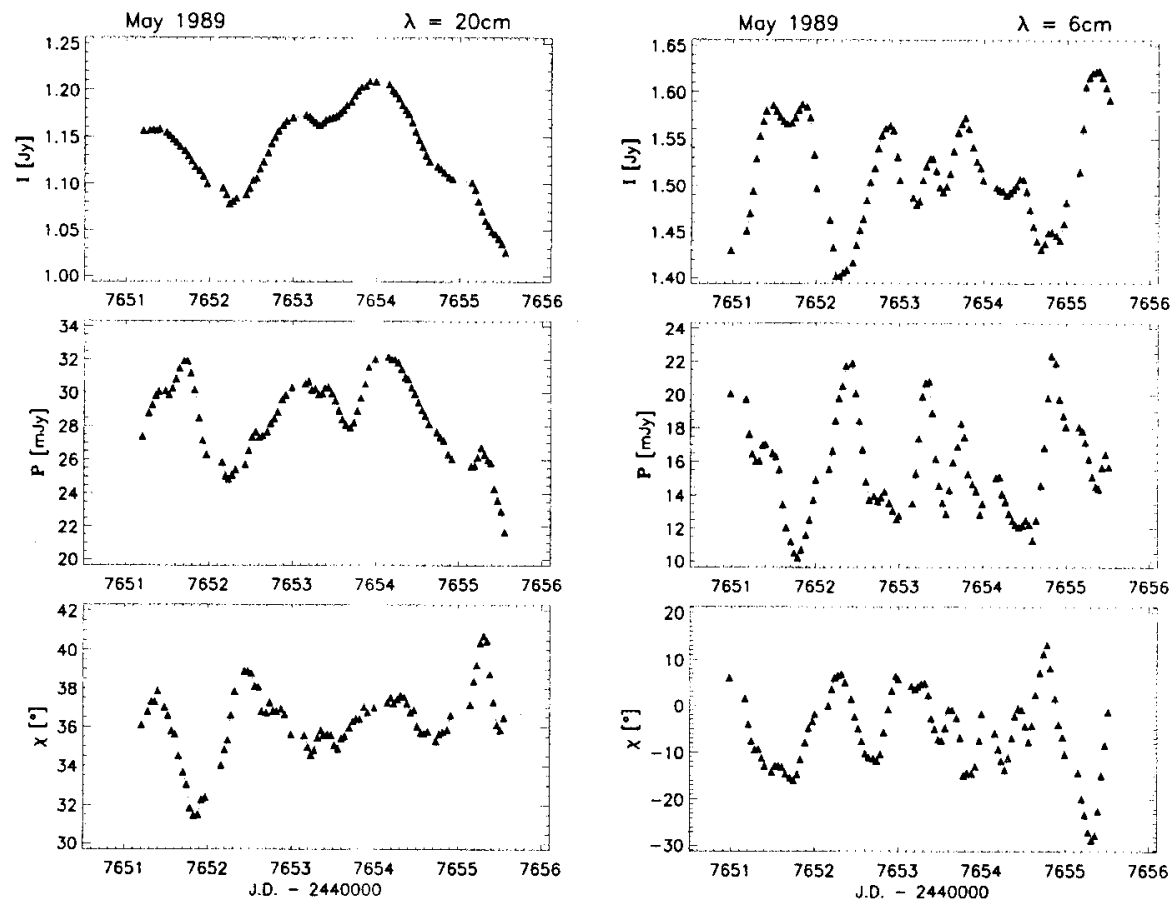

Figure 1. May89-event $(0917+624)$ : the observed light-curves of the total flux density $I$, polarized flux density $P$ and polarization angle $\chi$ at $20 \mathrm{~cm}$ and $6 \mathrm{~cm}$.

\section{Light Curves}

Multifrequency polarization observations for $0917+624$ were made in May 1989 at five wavelengths $(20,11,6,3.6$ and $2 \mathrm{~cm}$ ) using the VLA (Quirrenbach et al., 2000). As an example, the light curves of the total flux density, polarized flux density and polarization angle observed at 20 and $6 \mathrm{~cm}$ are shown in Figure 1 . Table I shows the mean values of the total flux density $(\bar{I})$, polarized flux density $(\bar{P})$, position angle $(\bar{\chi})$, polarization degree $(\bar{p})$ and the modulation indices $m_{l}$ and $m_{P}$. It can be seen that the polarization degrees $\bar{p}$ are low and $m_{P} \gg m_{l}$, implying more dramatic variations in polarization. Another interesting property is the increase of the mean polarization angle with wavelength.

\section{Polarization Properties}

It is found that the variations of the polarized flux density highly depend on wavelength. During the period JD 2447652-7655 (except JD 2447653.5-7654.0), the fractional variations of the polarized flux density at $20 \mathrm{~cm}$ are precisely proportional to those of the total flux density with a correlation coefficient of $\sim 97 \%$. The 


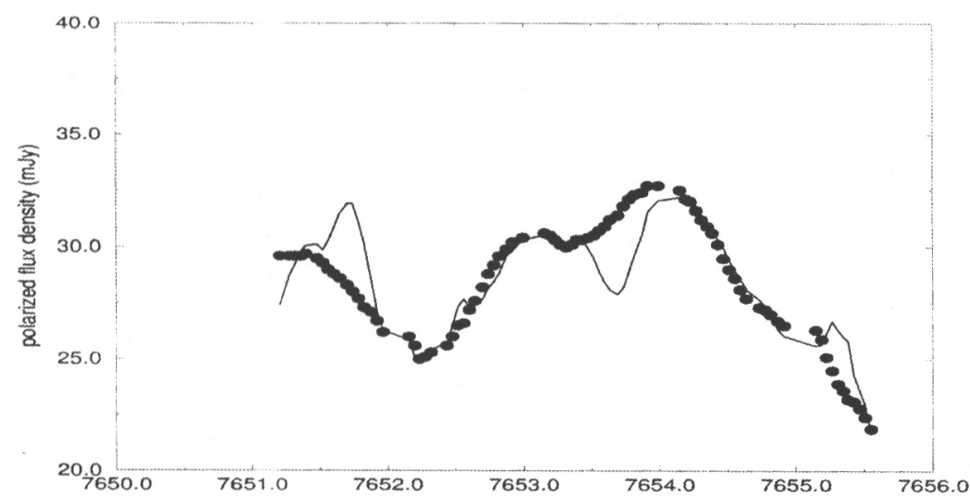

Figure 2. A model-fit to the variations of the $20 \mathrm{~cm}$ polarized flux density. Solid line - observation, points - model.

position angle was observed to be slightly variable. A two-component model with one scintillating component (Qian et al., in preparation) can well fit the proportional part of the polarized flux density light curve (Figure 2). But after subtracting the variations due to scintillation, three 'features' emerge, implying that an additional variable component is present. At $2-6 \mathrm{~cm}$, the relationship between the variations of the polarized and total flux density is more complex. For example at $6 \mathrm{~cm}$ (see Figure 1), both correlations and anti-correlations between the polarized and total flux density were observed. The transition between the two kinds of correlations was very rapid $(<0.5$ days, e.g. during the interval JD 2447652.57653.5).

\section{Correlation between $20 \mathrm{~cm}$ Features and Variations at 2-6 $\mathrm{cm}$}

As shown above, after subtracting the variations due to scintillation at $20 \mathrm{~cm}$, three residual features are separated at a significance level of $\sim 3 \sigma$. Interestingly they all coincide with the peaks in the $2-6 \mathrm{~cm}$ total flux light curves. The $20 \mathrm{~cm}$ features and the minibursts at $2-6 \mathrm{~cm}$ have almost the Same duration of $\sim 0.5$ days (Figure 3 ). This seems to imply that the $20 \mathrm{~cm}$ features and the $2-6 \mathrm{~cm}$ variations are $\mathrm{sim}$ ultaneous and caused by the same mechanism. In addition, the structure function of the $20 \mathrm{~cm}$ residual polarized flux is also similar to those of the total polarized flux densities at $2-6 \mathrm{~cm}$. This possible correlation and simultaneity may be useful to distinguish between intrinsic and extrinsic causes of IDV. More multifrequency polarization observations are desirable for confirmation. 

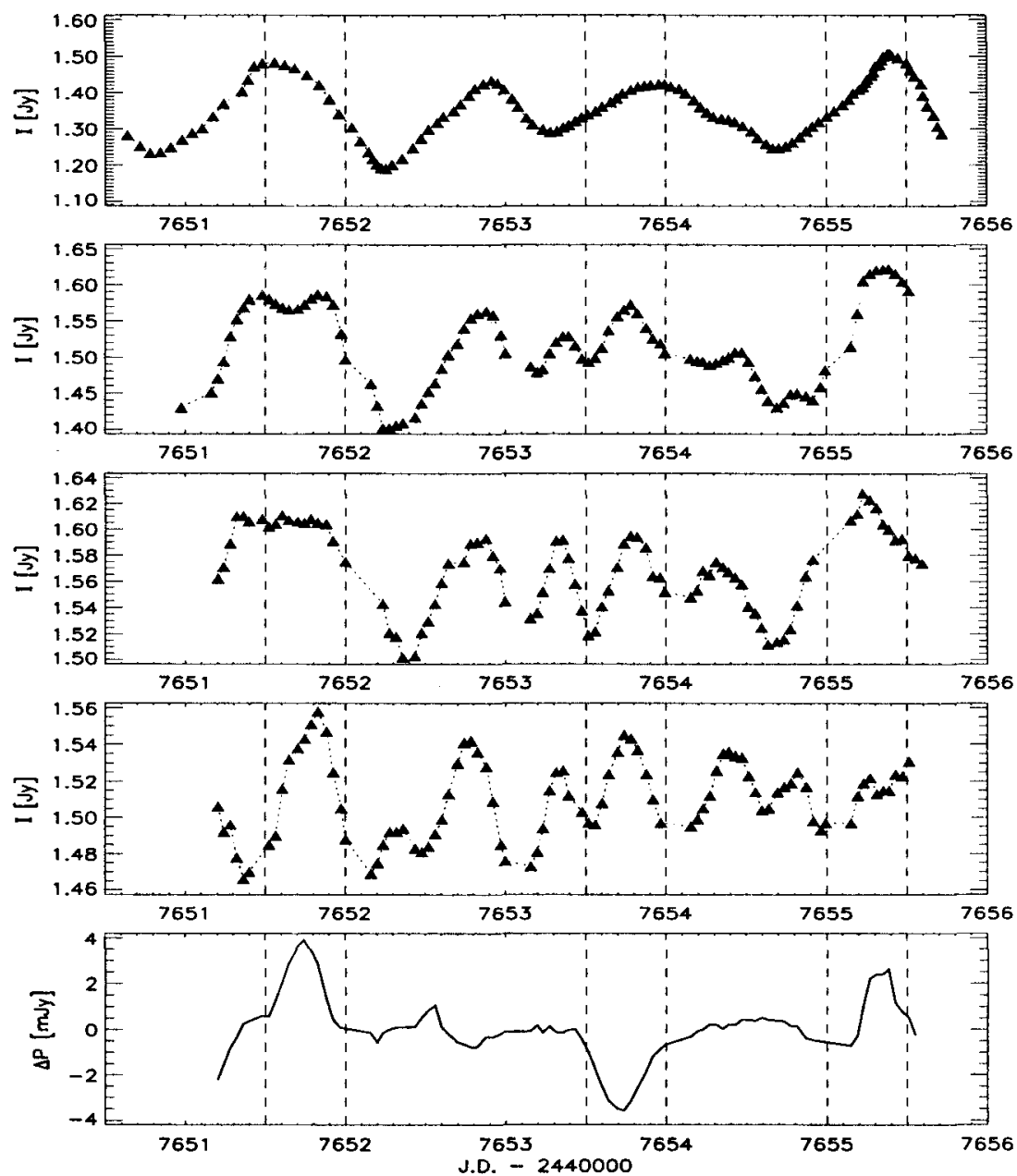

Figure 3. Correlation between the $20 \mathrm{~cm}$ residual polarized features and the total flux density variations at $2-6 \mathrm{~cm}$. From top to bottom: 11,6,3.6,2 and $20 \mathrm{~cm}$. The vertical dashed lines indicate the time-interval when the correlation may possibly occur.

\section{References}

Dennett-Thorpe, J. and de Bruyn, A.G.: 2000, ApJ 529, L65.

Gabuzda, D.C. and Kochanev, P.Yu.: 1997, vistas of Astronomy 41, 219.

Kedziora-Chudczer, L., Jauncey, D.L., Wieringa M.H., et al.: 1997, ApJ 490, L9.

Qian, S.J.: 1994a, AApS 14, 333 (Transl.: Chin. Astrora. 1995, 19, 69.)

Qian, S.J.: 1994b, AAnS 35, 362 (Transl.: Chin. Astrora. 1995, 19, 267.

Qian, S.J., Li, X.C., Wegner, R., et al.: 1996, Chin. Astrora. 20, 15.

Quirrenbach, A., Kraus, A., Witzel, A., et al.: 2000, A\&AS 141, 221.

Rickett, B.J., Quirrenbach, A., Wegner, R., et al.: 1995, A\&A 293, 479.

Wagner, S.J. and Witzel, A.: 1995, ARA\&A 33, 163. 